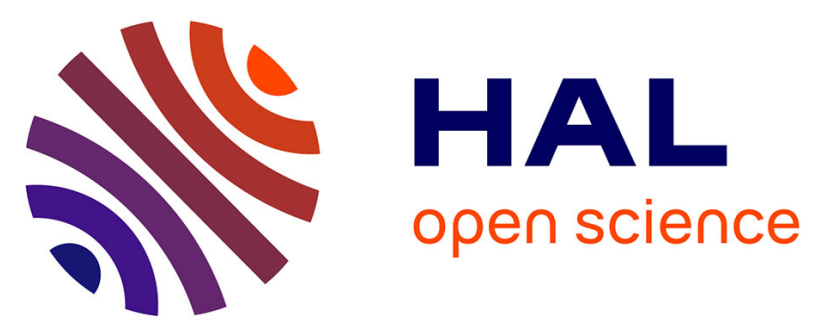

\title{
Le protocole de l'intervention mapping: un processus méthodique pour élaborer, implanter et évaluer des programmes en promotion de la santé
}

Jean-Baptiste Fassier, Marion Lamort-Bouché, Philippe Sarnin, Christine Durif-Bruckert, Julien Peron, Laurent Letrilliart, Marie-José Durand

\section{To cite this version:}

Jean-Baptiste Fassier, Marion Lamort-Bouché, Philippe Sarnin, Christine Durif-Bruckert, Julien Peron, et al.. Le protocole de l'intervention mapping: un processus méthodique pour élaborer, implanter et évaluer des programmes en promotion de la santé. Epidemiology and Public Health = Revue d'Epidémiologie et de Santé Publique, 2016, 64 (1), pp.33-44. 10.1016/j.respe.2015.10.002 . halshs-01426800

\author{
HAL Id: halshs-01426800 \\ https://shs.hal.science/halshs-01426800
}

Submitted on 10 Jul 2017

HAL is a multi-disciplinary open access archive for the deposit and dissemination of scientific research documents, whether they are published or not. The documents may come from teaching and research institutions in France or abroad, or from public or private research centers.
L'archive ouverte pluridisciplinaire HAL, est destinée au dépôt et à la diffusion de documents scientifiques de niveau recherche, publiés ou non, émanant des établissements d'enseignement et de recherche français ou étrangers, des laboratoires publics ou privés. 


\section{ScienceDirect}

www.sciencedirect.com
$\mathrm{EM} \mid$ consulte

www.em-consulte.com
Revue d'Épidémiologie et de Santé Publique

Revue d'Épidémiologie et de Santé Publique 64 (2016) 33-44

Article original

\title{
Le protocole de l'intervention mapping : un processus méthodique pour élaborer, implanter et évaluer des programmes en promotion de la santé
}

\section{The intervention mapping protocol: A structured process to develop, implement and evaluate health promotion programs}

\author{
J.-B. Fassier ${ }^{\text {a,*,b }}$, M. Lamort-Bouché ${ }^{\mathrm{a}, \mathrm{c}}$, P. Sarnin ${ }^{\mathrm{d}}$, C. Durif-Bruckert ${ }^{\mathrm{d}}$, J. Péron ${ }^{\mathrm{e}}$, \\ L. Letrilliart ${ }^{\mathrm{c}, \mathrm{f}}$, M.-J. Durand ${ }^{\mathrm{b}}$ \\ ${ }^{a}$ UMR T 9405, unité mixte de recherche épidémiologique et de surveillance transport travail environnement (UMRESTTE), université Claude-Bernard Lyon 1, 8, \\ avenue Rockefeller, 69373 Lyon cedex 08, France \\ ${ }^{\mathrm{b}}$ CAPRIT, campus Longueuil, université de Sherbrooke, 150, place Charles-Le Moyne, bureau 200, J4K 0A8 Longueuil, QC, Canada \\ ${ }^{\mathrm{c}}$ Département de médecine générale, université Claude-Bernard Lyon 1, 8, avenue Rockefeller, 69373 Lyon cedex 08, France \\ ${ }^{\mathrm{d}}$ EA 4163, institut de psychologie, groupe de recherche en psychologie sociale (GREPS), université Lyon 2, 5, avenue P.-Mendès-France, 69656 Bron, France \\ ${ }^{\mathrm{e}}$ UMR 5558, laboratoire de biométrie et biologie évolutive (LBBE), hôpital Lyon Sud, université Claude-Bernard Lyon 1, 165, chemin du Grand-Revoyet, 69495 \\ Pierre-Bénite, France \\ ${ }^{\mathrm{f}}$ EA 4129, santé individu société (SIS), institut de psychologie, université Lyon 2, 5, avenue P.-Mendès-France, 69676 Bron, France
}

Reçu le 12 avril 2015 ; accepté le 5 octobre 2015

Disponible sur Internet le 30 décembre 2015

\begin{abstract}
Background. - Health promotion programs are expected to improve population health and reduce social inequalities in health. However, their theoretical foundations are frequently ill-defined, and their implementation faces many obstacles. The aim of this article is to describe the intervention mapping protocol in health promotion programs planning, used recently in several countries.

Methods. - The challenges of planning health promotion programs are presented, and the six steps of the intervention mapping protocol are described with an example. Based on a literature review, the use of this protocol, its requirements and potential limitations are discussed.

Results. - The intervention mapping protocol has four essential characteristics: an ecological perspective (person-environment), a participative approach, the use of theoretical models in human and social sciences and the use of scientific evidence. It comprises six steps: conduct a health needs assessment, define change objectives, select theory-based change techniques and practical applications, organize techniques and applications into an intervention program (logic model), plan for program adoption, implementation, and sustainability, and generate an evaluation plan. This protocol was used in different countries and domains such as obesity, tobacco, physical activity, cancer and occupational health. Although its utilization requires resources and a critical stance, this protocol was used to develop interventions which efficacy was demonstrated.

Conclusion. - The intervention mapping protocol is an integrated process that fits the scientific and practical challenges of health promotion. It could be tested in France as it was used in other countries, in particular to reduce social inequalities in health.
\end{abstract}

(C) 2016 Published by Elsevier Masson SAS.

Keywords: Health promotion; Health planning; Program evaluation; Health status disparities

Résumé

Position du problème. - Les programmes en promotion de la santé sont supposés améliorer la santé de la population et réduire les inégalités sociales de santé. Cependant, leur développement repose souvent sur des bases théoriques insuffisantes et leur implantation se heurte à de

\footnotetext{
* Auteur correspondant.

Adresse e-mail : jean-baptiste.fassier@univ-lyon1.fr (J.B. Fassier), marion.lamort-bouche@univ-lyon1.fr (M. Lamort-Bouché), philippe.sarnin@univ-lyon2.fr (P. Sarnin), christine.durif@univ-lyon2.fr (C. Durif-Bruckert), julien.peron@chu-lyon.fr (J. Péron), laurent.letrilliart@wanadoo.fr (L. Letrilliart), marie-jose.durand@usherbrooke.ca (M.J. Durand).
} 
nombreux obstacles. L'objectif de cet article est de décrire le protocole de l'intervention mapping de planification de programmes en promotion de la santé, utilisé récemment dans plusieurs pays.

Méthodes. - Les enjeux de la planification des programmes en promotion de la santé sont présentés et les six étapes du protocole de l'intervention mapping sont décrites avec un exemple. À partir d'une revue de la littérature, différentes utilisations et les contraintes de ce protocole sont discutées, ainsi que ses limitations possibles.

Résultats. - Le protocole de l'intervention mapping présente quatre caractéristiques essentielles : la perspective écologique (personneenvironnement), la participation des acteurs, le recours aux cadres théoriques appropriés en sciences humaines et sociales et le recours aux données scientifiques valides. Il comprend six étapes : conduire une évaluation des besoins, formuler des objectifs de changement, choisir les théories appropriées en sciences humaines et sociales et les méthodes d'intervention, développer le modèle logique de programme, planifier l'adoption, l'implantation et la pérennisation du programme, et planifier son évaluation. Ce protocole a été utilisé dans plusieurs pays et dans des domaines aussi variés que la lutte contre l'obésité, le tabagisme, la sédentarité, le cancer et dans le domaine de la santé au travail. Bien que son utilisation soit contraignante et nécessite un regard critique, il a permis de développer plusieurs interventions dont l'efficacité a été documentée.

Conclusion. - Le protocole de l'intervention mapping est un processus permettant de répondre aux enjeux scientifiques et pratiques de la promotion de la santé. Utilisé dans d'autres pays, cet outil pourrait être testé en France, notamment pour réduire les inégalités sociales de santé. (C) 2016 Publié par Elsevier Masson SAS.

Mots clés : Promotion de la santé ; Planification en santé ; Évaluation de programmes ; Disparités de l'état de santé

\section{Introduction}

L'augmentation des maladies chroniques liées aux modes de vie a conduit au développement de la promotion de la santé pour modifier les comportements et les environnements et ainsi améliorer l'état de santé de la population [1]. La promotion de la santé vise également à réduire les inégalités sociales de santé qui ont augmenté ces dernières décennies malgré la progression de l'espérance de vie de la population générale [2]. Le développement de programmes en promotion de la santé est censé améliorer l'état de santé de la population et réduire les inégalités, mais il se heurte à de nombreuses barrières [3]. Le protocole de l'intervention mapping $^{1}[4,5]$ a été utilisé dans plusieurs pays depuis une quinzaine d'années pour différentes problématiques de santé publique telles que la lutte contre l'obésité, le tabagisme, la sédentarité ou le cancer. Cet article a pour objectifs de décrire les enjeux de la planification de programmes en promotion de la santé (Section 2), de présenter le protocole de l'intervention mapping (Section 3), de décrire ses différentes étapes illustrées par un exemple (Section 4) et de discuter les enjeux associés à l'utilisation de ce protocole (Section 5).

\section{La promotion de la santé}

\subsection{Cadre général}

La promotion de la santé s'est progressivement développée comme un champ de pratique et de recherche spécifique au sein de la santé publique [6]. Elle est définie dans la charte d'Ottawa de l'Organisation mondiale de la santé (OMS) comme «le processus qui confère aux populations les moyens d'assurer un

\footnotetext{
${ }^{1}$ Il n'existe pas de traduction simple de l'expression « intervention mapping » qui correspond à la recherche, l'analyse, la hiérarchisation et la mise en relation des concepts permettant de décrire un problème de santé et l'intervention à développer, implanter puis évaluer pour y remédier. La traduction littérale en français ( « cartographie d'intervention ») pouvant prêter à confusion, le choix a été fait de conserver l'expression princeps en anglais.
}

plus grand contrôle sur leur propre santé et d'améliorer celleci ». [1] La promotion de la santé s'inscrit dans une vision globale de la santé et de ses déterminants, qu'ils soient situés au niveau des individus, de l'environnement ou de l'organisation du système de soins de santé.

Les déterminants individuels comportent des facteurs biologiques ainsi que les comportements. Les comportements en matière de santé sont définis comme toutes les activités qu'un individu peut entreprendre pour promouvoir, protéger ou maintenir sa santé [7]. Il s'agit par exemple d'avoir une activité physique régulière, une alimentation équilibrée, se faire vacciner ou se faire dépister (frottis cervico-utérin, dépistage du cancer du côlon, etc.). Les comportements à risque sont ceux qui sont associés à une vulnérabilité accrue à l'égard d'une maladie déterminée (par exemple la consommation excessive d'alcool et de tabac favorisant le cancer des voies aérodigestives supérieures) [7]. Les comportements de santé ont fait l'objet de nombreuses études en psychologie de la santé et en psychologie sociale [8-10]. Différents concepts ont été progressivement développés, tels que la notion de vulnérabilité et de sévérité perçues envers la maladie, les coûts et les bénéfices attendus après l'adoption d'un comportement, le sentiment d'auto-efficacité, la croyance et l'adhésion aux normes sociales de l'environnement, etc. Ces concepts ont été intégrés dans des modèles tels que le modèle des croyances en matière de santé [11], la théorie du comportement planifié [12], la théorie du comportement raisonné, la théorie sociocognitive [13,14], le modèle trans-théorique des stades de changement [15], le modèle de précaution-adoption [16] ou encore d'autres théories [10]. Ces modèles explicatifs adoptent une perspective essentiellement individuelle sur l'adoption des comportements. Le rôle de l'environnement y est rarement opérationnalisé par des indicateurs mesurables. Il s'agit de l'environnement perçu par un individu (norme sociale perçue, par exemple) plutôt que d'éléments factuels de cet environnement. Pourtant, l'adoption d'un comportement ne se limite pas à la seule responsabilité individuelle et peut être déterminée par l'environnement, y compris l'environnement passé [2,17,18]. 
Les déterminants sociaux de la santé ont été catégorisés dans une typologie proposée par l'OMS [19]. Les déterminants structurels appartiennent au contexte politique et socioéconomique du pays. Ils ont un impact sur la distribution inégale des déterminants intermédiaires, qui renvoient aux conditions matérielles (logement, environnement géographique, environnement physique de travail) et aux facteurs psychosociaux (stress des conditions de vie et de travail, relations et soutien social).

Enfin, les déterminants de la santé sont situés au niveau du système de soins, avec des disparités territoriales (démographie médicale, équipement, offre de soins) et des barrières socioculturelles et économiques vis-à-vis de l'accès aux soins médicaux ou de prévention. Ces constats interrogent la capacité des politiques de santé et de l'organisation du système de santé à répondre aux besoins des différentes catégories de la population, plus particulièrement celles défavorisées sur un plan socioéconomique. Ils invitent également à imaginer une offre innovante de soins et de services pour rejoindre ces populations et réduire les inégalités sociales de santé [20,21]. Selon la Charte d'Ottawa, « l'effort de promotion de la santé vise à l'équité en matière de santé » dans le «but (...) de réduire les écarts actuels dans l'état de santé ». Pour autant, la planification de programmes en promotion de la santé doit relever de nombreux défis.

\subsection{Planification de la santé}

La planification de la santé est définie comme un processus continu de prévision de ressources et de services requis pour atteindre des objectifs déterminés [22]. Elle comprend successivement l'évaluation des besoins de santé, la détermination des priorités, la conception des interventions, leur mise en œuvre et leur évaluation [22]. En France, les deux premières étapes correspondent à l'élaboration des plans régionaux de santé au niveau de chaque agence régionale de santé. Plusieurs guides méthodologiques ont été publiés pour faciliter la tâche des acteurs à ce stade $[23,24]$. Cependant, la conception et la mise en œuvre des interventions ne disposent pas actuellement de telles orientations méthodologiques. Il est reproché à de nombreuses interventions de ne pas être étayées sur des fondements théoriques suffisants, ni de bénéficier d'une évaluation adéquate, que ce soit en France [3] ou à l'étranger [25]. Ces lacunes ont été attribuées notamment à une intégration insuffisante des concepts et des méthodes de recherche sur les programmes de santé [26,27].

Les programmes en promotion de la santé présentent toutes les caractéristiques des interventions complexes « faisant partie d'un système organisé d'action visant à agir sur des problèmes divergents dans un contexte donné, au cours du temps » [28]. Cette complexité est générée par la difficulté des changements de comportements, le nombre de groupes visés, le nombre de critères d'intérêt et la flexibilité nécessaire des interventions [29,30]. La conception de ces interventions relève de méthodes particulières de recherche issues de la recherche évaluative [26] et de la recherche interventionnelle en santé des populations [27]. L'analyse logique, par exemple, correspond à la phase d'élaboration du contenu et des modalités de l'intervention.
Elle évalue selon des méthodes déterminées le bien-fondé du modèle logique théorique (hypothèses de l'efficacité et principes d'actions) et du modèle logique opérationnel (modalités pratiques) de l'intervention [26,31,32]. La participation des groupes de population auxquelles l'intervention est destinée et des acteurs qui délivrent cette intervention est nécessaire, mais difficile à obtenir et maintenir. À ce titre, ces programmes doivent être considérés comme des innovations complexes avec une probabilité importante d'échec de l'implantation et/ou de la pérennisation [33]. Ces éléments impliquent de considérer soigneusement leur stratégie d'implantation [30,34-36].

En synthèse, la planification des programmes en promotion de la santé nécessite une vision globale des déterminants de la santé et la maîtrise des méthodes de recherche évaluative et de recherche interventionnelle en santé des populations. Le protocole de l'intervention mapping utilisé depuis une quinzaine d'années a été pensé pour intégrer cette complexité et guider les concepteurs de programmes dans ces enjeux à travers une démarche structurée.

\section{Le protocole de l'intervention mapping : histoire et caractéristiques}

\subsection{Contexte historique}

Le protocole de l'intervention mapping est une méthode de planification des programmes en promotion de la santé mise en œuvre à la fin des années 1990 [4,37]. Il a été développé dans la continuité d'autres approches en promotion de la santé, et plus particulièrement le modèle PRECEDE de Green et Kreuter [38] et l'approche du marketing social [39]. Il s'inscrit également dans certaines approches d'évaluation de programmes comme l'évaluation guidée par la théorie [40,41] et l'évaluation réaliste [42]. Il a été reproché à ces méthodes de considérer uniquement l'évaluation des besoins (modèle PRECEDE), ou encore l'évaluation des interventions seulement a posteriori, aboutissant dans les deux cas à l'absence d'orientation pour élaborer en amont le contenu des interventions [37].

\subsection{Caractéristiques essentielles}

Le protocole présente quatre caractéristiques essentielles :

- la formulation des problèmes dans une perspective écologique ;

- la participation des acteurs concernés ;

- le recours aux cadres théoriques appropriés en sciences humaines et sociales ;

- le recours aux données scientifiques valides [4,37].

\subsubsection{Perspective écologique}

Elle implique de considérer l'ensemble des comportements individuels au sein des différents niveaux d'environnement dans lequel ils interviennent : environnement conjugal, familial, de voisinage, professionnel, sociétal, etc. Cette perspective correspond à la vision globale de la santé et de ses déterminants évoquée précédemment. 


\subsubsection{Approche participative}

Plusieurs parties prenantes ayant des intérêts parfois divergents sont impliquées dans les problématiques en promotion de la santé. Les groupes de population auxquels l'intervention est destinée, les acteurs de leur environnement (famille, voisinage, milieu de travail, etc.), les acteurs qui financent et ceux qui délivrent cette intervention, et enfin les chercheurs qui l'évaluent. Le protocole prévoit la constitution d'un groupe de travail collaboratif permettant de représenter cette diversité d'intérêts et d'accompagner les planificateurs à chacune des étapes. Cette participation est invoquée au motif d'augmenter la pertinence de l'intervention développée, sa légitimité, son acceptation et finalement sa pérennité. Elle procède aussi de la perspective écologique visant à modifier les différents niveaux d'environnement impliqués. La participation des populations visées par l'intervention (parfois appelées «populations cibles » ou «populations prioritaires ») est particulièrement importante aux étapes 1 (évaluation des besoins), 4 (finalisation) et 5 (adoption et implantation).

\subsubsection{Recours aux cadres théoriques en sciences humaines et sociales}

L'identification des déterminants comportementaux et environnementaux du problème de santé implique de recourir à différents cadres théoriques pour comprendre et influencer ces déterminants à chaque niveau considéré : psychologique (individuel), interpersonnel, organisationnel, communautaire, politique, sociétal, etc. Un résumé synthétique de ces théories a été établi par le National Cancer Institute aux États-Unis [43]. Le Tableau 1 en représente une synthèse non exhaustive.

\subsubsection{Recours aux données scientifiques valides}

Chaque étape du protocole soulève des questions différentes nécessitant une recherche bibliographique et l'évaluation critique des données scientifiques publiées, s'agissant par exemple des déterminants des problèmes de santé et de l'efficacité des méthodes pour modifier les déterminants des comportements et de l'environnement. Différents outils d'évaluation critique de la littérature sont utilisables selon la nature des études considérées (études observationnelles, essais randomisés, études qualitatives, méta-analyses, etc.) et sont accessibles sur Internet (http://www. equator-network.org/).

\section{Le protocole de l'intervention mapping : différentes étapes}

Ce protocole propose six étapes pour guider les planificateurs :

- conduire une évaluation des besoins ;

- formuler les objectifs du programme ;

- choisir les théories et les méthodes d'intervention ;

- développer le modèle logique du programme ;

- planifier l'adoption et l'implantation du programme ;

- planifier son évaluation.

Ces différentes étapes et leur contenu sont illustrés concrètement dans les sections suivantes à partir du programme
AMIGAS de dépistage du cancer du col utérin (voir Encadré 1) $[44,45]$.

\section{1. Évaluation des besoins}

La première étape vise à obtenir une formulation complète du problème de santé (et/ou de qualité de vie). L'évaluation des besoins procède par méthodes mixtes. Une approche quantitative par indicateurs est destinée à formuler une vision épidémiologique du problème (mortalité, morbidité, ou consommation de soins, etc.) [22]. Parallèlement, l'évaluation des besoins dans la perspective des parties prenantes est recueillie selon différentes modalités qualitatives (entrevues, focus groupes) ou quantitatives (enquêtes). C'est le croisement de ces deux visions qui permet de produire une représentation du problème fondée à la fois sur les savoirs techniques des gestionnaires et des praticiens du système de santé, et sur les savoirs d'expérience des personnes confrontées au problème.

La vision écologique des différents déterminants de la santé est particulièrement importante à ce stade de la définition du problème car elle va orienter la recherche des solutions. Elle postule que la responsabilité du changement n'incombe pas seulement aux individus ayant un problème de santé, mais aussi aux différentes structures sociales dans lesquelles ils vivent (famille, quartier, milieu de travail, etc.). C'est pourquoi la formulation (théorie) du problème ne se limite pas à l'analyse des comportements de santé individuels dans la population visée mais comprend également leur environnement (comportement des acteurs de l'environnement).

L'approche participative est également prépondérante à cette étape. Une attention particulière est consacrée à la constitution d'un groupe de travail collaboratif (comité scientifique élargi) associant les chercheurs avec les différentes parties prenantes, dont les représentants de la population à laquelle l'intervention est destinée. Le processus de consultation est crucial pour identifier les déterminants à l'origine des comportements qui doivent être modifiés, autant chez les individus dans la population cible que chez les acteurs dans leur environnement.

Les déterminants à l'origine des comportements sont assimilables aux « causes racines » du problème, parfois appelées « causes des causes ». Dans le modèle théorique PRECEDE [46] sur lequel la première étape du protocole est fondée, ces déterminants sont catégorisés en facteurs prédisposant, facilitant, et de renforcement. Dans le protocole de l'intervention mapping, ces déterminants sont rapprochés des concepts clés en psychologie sociale et de la santé (connaissances, compétences, sentiment d'efficacité personnelle, attentes de résultats, norme sociale perçue, etc.) afin d'identifier les théories les mieux adaptées pour expliquer la problématique. Au terme de cette première étape, les parties prenantes doivent s'accorder sur la formulation (théorie) du problème, incluant le choix des objectifs distaux attendus de l'intervention à développer (indicateurs de santé, de qualité de vie ou autres). 
Tableau 1

Principaux modèles, niveaux et concepts clés des théories mobilisables.

\begin{tabular}{|c|c|c|}
\hline Théorie & Focus & Concepts clés \\
\hline \multicolumn{3}{|l|}{ Théorie de niveau individuel } \\
\hline Modèle des croyances de santé [11] & $\begin{array}{l}\text { Perceptions par un individu des menaces liées à un problème } \\
\text { de santé, du bénéfice à éviter cette menace et facteurs } \\
\text { influençant la décision d'agir }\end{array}$ & $\begin{array}{l}\text { Susceptibilité perçue } \\
\text { Sévérité perçue } \\
\text { Bénéfices perçus } \\
\text { Barrières perçues } \\
\text { Clés pour agir } \\
\text { Efficacité personnelle }\end{array}$ \\
\hline Modèle des stades de changements [15] & $\begin{array}{l}\text { Motivation d'un individu et niveau de préparation à changer } \\
\text { son comportement par rapport à un problème }\end{array}$ & $\begin{array}{l}\text { Pré-contemplation } \\
\text { Contemplation } \\
\text { Décision } \\
\text { Action } \\
\text { Maintien }\end{array}$ \\
\hline Théorie du comportement planifié [12] & $\begin{array}{l}\text { Attitudes d'un individu envers un comportement, } \\
\text { perceptions des normes, et croyances sur la facilité ou la } \\
\text { difficulté de changer }\end{array}$ & $\begin{array}{l}\text { Intention de comportement } \\
\text { Attitude } \\
\text { Norme subjective } \\
\text { Contrôle comportemental perçu }\end{array}$ \\
\hline Modèle du processus précaution-adoption [16] & $\begin{array}{l}\text { Trajectoire d'un individu depuis le manque de conscience } \\
\text { jusqu'à l'action et le maintien }\end{array}$ & $\begin{array}{l}\text { Inconscient du problème } \\
\text { Non concerné par le problème } \\
\text { Décidé à ne pas agir } \\
\text { Décidé à agir } \\
\text { Action } \\
\text { Maintien }\end{array}$ \\
\hline
\end{tabular}

Théorie de niveau interpersonnel

Théorie sociale cognitive [14]

Théories de niveau communautaire

Organisation communautaire

Diffusion des innovations [33]

Théorie de la communication
Les facteurs personnels, de l'environnement, et le comportement humain s'influencent mutuellement

Approche dirigée par la communauté pour évaluer et résoudre les problèmes de santé et les problèmes sociaux

Comment les idées nouvelles, les produits et les pratiques s'étendent dans une société ou d'une société à l'autre

Comment les différents types de communication affectent les comportements de santé
Déterminisme réciproque Capacité comportementale Attentes

Efficacité personnelle

Apprentissage par observation

Renforcement

\section{Empowerment}

Capacité communautaire Participation

Pertinence

Sélection des problèmes

Conscience critique

Avantages relatifs

Compatibilité

Complexité

Possibilité d'essayer (trialability)

Visibilité

Exemple Agenda setting

Agenda médiatique

Agenda public

Agenda politique

Identification et définition du problème

D'après [43].

Dans le cadre du programme AMIGAS [45], une liste des déterminants conduisant à la réalisation d'un frottis cervico-utérin (FCU) a été établie suite aux focus groupes et enquêtes conduites dans la population concernée. Secondairement, ces déterminants ont été catégorisés en facteurs prédisposant, facilitant et de renforcement selon le modèle PRECEDE [38,46] et reliés aux modèles théoriques en psychologie de la santé (voir Encadré 1).

\subsection{Formulation des objectifs de changement}

La deuxième étape consiste à formuler les objectifs chiffrés des résultats attendus de l'intervention à développer, à partir du modèle logique du problème précédemment élaboré. On distingue 3 catégories d'objectifs selon leur temporalité :

- les objectifs distaux (finaux) sont les effets attendus du programme sur l'état de santé et/ou la qualité de vie ;

- les objectifs de performance (intermédiaires) sont les effets attendus du programme sur les comportements dans la population cible et/ou les acteurs de l'environnement;

- les objectifs de changement (proximaux) sont les effets attendus du programme sur les déterminants des comportements (connaissances, compétences, efficacité personnelle, norme sociale perçue, attente de résultats, etc.). 
Encadré 1. Développement du programme AMIGAS au moyen du protocole de l'intervention mapping

Ce programme de dépistage du cancer du col utérin a été développé au Texas et subventionné par le Center for Disease Control and Prevention pour les femmes hispanophones vivant le long de la frontière mexicaine. Ce groupe de population avait été identifié comme ayant un dépistage plus limité et une morbidité supérieure au reste de la population s'agissant du cancer du col utérin. Pour développer ce programme en suivant les étapes du protocole de l'intervention mapping, les auteurs ont répondu aux questions suivantes, aux étapes 1 et 3 du protocole.

Étape 1 de l'élaboration du programme : évaluer les besoins et formuler une théorie du problème

Quels sont les déterminants qui influencent la réalisation d'un frottis cervico-utérin (FCU) dans ce groupe de population ?

Selon la terminologie du modèle PRECEDE, les auteurs ont distingué les facteurs prédisposant (susceptibilité perçue envers le cancer du col utérin, bénéfices perçus du dépistage, niveau de connaissance sur la maladie, sentiment d'efficacité personnelle), les facteurs facilitants (accès ou barrières perçues au dépistage, statut d'immigrant) et les facteurs de renforcement (soutien du conjoint ou du médecin, récompense personnelle).

Quels sont les cadres théoriques les plus appropriés pour intégrer ces déterminants en vue d'expliquer ou de prédire la réalisation d'un FCU?

Les auteurs ont identifié quatre cadres théoriques principaux : le modèle des croyances de santé, la théorie de l'action raisonnée, le modèle trans-théorique du changement et la théorie sociale cognitive.

Étape 3 de l'élaboration du programme : sélectionner les théories et les techniques d'intervention

Quelles techniques de changement et modalités pratiques utiliser pour modifier les déterminants qui influencent la réalisation d'un FCU dans ce groupe de population?

Les techniques de changement identifiées par les auteurs étaient la délivrance d'informations, la communication persuasive, I'observation, I'apprentissage de nouvelles compétences, la pratique guidée avec une rétro-information, le renforcement social et le renforcement vicariant. Les déclinaisons pratiques comportaient de multiples supports : un roman vidéo avec des acteurs de la communauté hispanophone (à passer sur un lecteur DVD portable), un cahier imagé reprenant les messages de la vidéo, une liste des établissements et indications pratiques pour faire pratiquer un FCU (horaires, transports), un spéculum et une brosse à frottis, un jeu à pratiquer en groupe, des cartes avec des messages, deux schémas représentant I'appareil génital féminin et la réalisation d'un FCU, et une feuille intitulée " ma promesse " représentant l'engagement symbolique dans les différentes étapes pour obtenir un FCU. Certains supports de ce programme sont consultables sur Internet (http://www.cdc.gov/cancer/gynecologic/ what_cdc_is_doing/amigas.htm).
C'est à ce stade que des objectifs spécifiques aux différents sous-groupes de population peuvent être formulés si des besoins différenciés ont été identifiés durant l'évaluation des besoins. Une attention particulière est portée à la définition précise des objectifs de performance (changements attendus des comportements), souvent déclinés en étapes intermédiaires s'agissant des comportements complexes.

Dans le cadre du programme AMIGAS, huit objectifs de performance avaient été définis :

- décider de réaliser un FCU ;

- identifier une structure où le réaliser ;

- prendre rendez-vous ;

- venir à son rendez-vous ;

- prendre connaissance de ses résultats ;

- prendre un autre rendez-vous si nécessaire ;

- venir au rendez-vous de suivi ;

- programmer un nouveau test à la date prévue.

La spécification des déterminants de chaque comportement procède par l'élaboration d'une série de matrices dont un exemple est donné dans le Tableau 2 . Il y est précisé ce qui était attendu des participantes au programme AMIGAS pour chaque déterminant en vue de parvenir à l'objectif de performance.

\subsection{Choix des cadres théoriques et de leurs applications pratiques}

La troisième étape est la sélection des théories et des techniques d'intervention permettant d'atteindre les objectifs de changement précédemment formulés. En premier lieu intervient le choix des cadres théoriques appropriés aux objectifs de changement, au contexte et à la population concernés. S'agissant des comportements individuels, les cadres théoriques sont le plus souvent issus de la psychologie de la santé et/ou de la psychologie sociale. Pour les changements dans l'environnement, des cadres théoriques appropriés à chaque niveau écologique sont recherchés autant que nécessaire dans le domaine de l'action communautaire, de la gestion, des sciences politiques, de la diffusion des innovations, de la communication, etc. [43].

Cette étape nécessite une expertise spécifique dans l'exploration des représentations et le changement des comportements, permettant de fonder les composantes d'une intervention multi-niveaux sur des assises théoriques solides. Il s'agit en quelque sorte de choisir soigneusement les «principes actifs » des composantes de l'intervention dont l'efficacité a déjà été documentée dans la littérature. Une fois les cadres théoriques identifiés, les techniques pratiques de changement comportemental sont choisies : il s'agit de la déclinaison concrète de ces théories sous la forme par exemple de modèles auxquels s'identifier, de jeux sérieux, de pratique guidée, etc. Le produit de cette troisième étape est le modèle logique théorique du programme, c'est-à-dire les mécanismes par lesquels il est supposé atteindre ses objectifs.

Dans le cadre du programme AMIGAS, les techniques de changement ont été choisies en fonctions des déterminants influençant la réalisation d'un FCU (voir Encadré 1). 
Tableau 2

Exemple de matrice de changement du programme AMIGAS (étape 2 du protocole). Comportement visé : dépistage par frottis cervico-utérin (FCU).

\begin{tabular}{|c|c|c|c|c|c|c|c|c|}
\hline \multirow{2}{*}{$\begin{array}{l}\text { Objectifs de } \\
\text { performance }\end{array}$} & \multicolumn{5}{|c|}{ Déterminants personnels ${ }^{\mathrm{a}}$} & \multicolumn{3}{|c|}{ Déterminants externes ${ }^{a}$} \\
\hline & Connaissances & $\begin{array}{l}\text { Attentes de } \\
\text { résultats }\end{array}$ & $\begin{array}{l}\text { Barrières et } \\
\text { bénéfices } \\
\text { perçus }\end{array}$ & Attitude & $\begin{array}{l}\text { Efficacité } \\
\text { personnelle }\end{array}$ & $\begin{array}{l}\text { Normes } \\
\text { sociales } \\
\text { perçues }\end{array}$ & $\begin{array}{l}\text { Soutien des } \\
\text { pairs/de } \\
\text { la famille }\end{array}$ & $\begin{array}{l}\text { Disponibilité } \\
\text { et accessibilité }\end{array}$ \\
\hline \multirow[t]{2}{*}{$\begin{array}{l}\text { Réaliser un } \\
\text { FCU au } \\
\text { moins tous } \\
\text { les trois ans }\end{array}$} & $\begin{array}{l}\text { Discute sur } \\
\text { ce qu'est le } \\
\text { cancer, et } \\
\text { pourquoi c'est } \\
\text { une menace }\end{array}$ & $\begin{array}{l}\text { S'attend à ce } \\
\text { que la réalisation } \\
\text { régulière d'un } \\
\text { FCU résultera } \\
\text { dans de meilleures } \\
\text { chances de survie } \\
\text { au cancer si elle } \\
\text { en développe un }\end{array}$ & $\begin{array}{l}\text { Exprime plus } \\
\text { de bénéfices } \\
\text { que de } \\
\text { barrières à } \\
\text { l'obtention } \\
\text { d'un FCU }\end{array}$ & $\begin{array}{l}\text { Croit que le } \\
\text { traitement } \\
\text { peut être } \\
\text { efficace pour } \\
\text { guérir d'un } \\
\text { cancer du col }\end{array}$ & $\begin{array}{l}\text { Exprime sa } \\
\text { confiance à } \\
\text { obtenir un } \\
\text { FCU }\end{array}$ & $\begin{array}{l}\text { Croit que les } \\
\text { autres femmes } \\
\text { comme elles } \\
\text { réalisent un } \\
\text { FCU }\end{array}$ & $\begin{array}{l}\text { Demande à } \\
\text { une amie ou } \\
\text { un membre } \\
\text { de la famille de } \\
\text { l'accompagner } \\
\text { à son RV }\end{array}$ & $\begin{array}{l}\text { La clinique } \\
\text { facilite la } \\
\text { prise du } \\
\text { rendez-vous }\end{array}$ \\
\hline & $\begin{array}{l}\text { Décrit les } \\
\text { recommandations } \\
\text { sur le dépistage } \\
\text { par FCU }\end{array}$ & & & $\begin{array}{l}\text { Décrit l'inconfort } \\
\text { ou l'embarras du } \\
\text { test comme minime } \\
\text { et/ou supportable }\end{array}$ & & & & \\
\hline
\end{tabular}

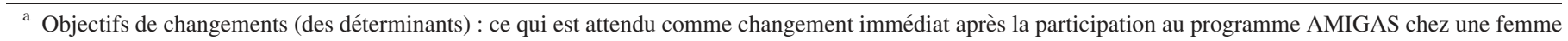
(déterminants personnels) ou les acteurs de son environnement (déterminants externes).

\section{4. Élaboration du programme}

La quatrième étape consiste à intégrer les techniques de changement choisies à l'étape précédente sous la forme d'un modèle logique du programme [26]. Ce modèle comprend le modèle logique théorique précédemment élaboré (hypothèses sous-jacentes permettant d'énoncer les objectifs et les mécanismes d'action du programme), et le modèle logique opérationnel comprenant le modèle d'organisation (qui va délivrer l'intervention, pendant combien de temps, avec quels moyens ?) et le modèle d'utilisation (à quel moment et à quel endroit les personnes auxquelles le programme est destiné vontelles être rejointes ?) [26,32]. La participation des représentants de la population visée est essentielle à ce stade, pour vérifier la pertinence, l'acceptabilité et la faisabilité du programme proposé. Les différents matériels du programme doivent être testés avant leur diffusion à plus grande échelle pour vérifier leur compréhension (supports d'information, procédures d'auto-soins, etc.).

Dans le cadre du programme AMIGAS, l'intervention était conçue pour être délivrée sur une base individuelle, ou bien en groupe. Dans les deux cas, elle était délivrée par des femmes de la communauté hispanophone spécialement formées (Promotoras) et disposant de supports multiples (voir Encadré 1).

\subsection{Adoption et implantation}

La cinquième étape vise à garantir l'implantation du programme et son adoption par les différentes parties prenantes. On distingue les participants au programme (appartenant à la population cible), les acteurs de l'environnement dont certains comportements doivent être modifiés, et les professionnels qui délivrent le programme. Il s'agit par exemple des professionnels de santé délivrant une intervention ou des membres d'une administration dont l'action est nécessitée par le programme. Des objectifs chiffrés d'implantation sont formulés, ainsi que des stratégies permettant de les atteindre dans les différentes catégories d'utilisateurs.

L'approche participative est importante à ce stade. Plusieurs échecs de l'implantation ont été décrits faute d'avoir anticipé les barrières individuelles et organisationnelles, et faute d'avoir associé les participants au programme et les professionnels le délivrant. Il est recommandé de considérer soigneusement le contexte d'adoption d'une nouvelle intervention pour élaborer une stratégie d'implantation appropriée aux différentes catégories d'acteurs dans les différents niveaux de l'environnement $[30,35]$.

Dans le cadre du programme AMIGAS, le recrutement des participantes s'est fait par la distribution de prospectus et par recrutement direct dans différents lieux fréquentés par les femmes hispanophones : salons de beauté, magasins de bijoux, épiceries, écoles, centres communautaires, églises, magasins de détail et laveries automatiques [44]. Après une première phase de test de deux ans, les supports du programme ont été affinés pour une meilleure compréhension par le public ciblé. La formation pendant trois jours des Promotoras dans chaque bassin de population faisait partie intégrante de la stratégie d'implantation du programme.

\section{6. Évaluation}

La sixième et dernière étape vise à évaluer l'intervention qui a été développée et implantée. Il s'agit le plus souvent d'une étude d'épidémiologie interventionnelle évaluant l'efficacité du programme au moyen du plan expérimental le plus approprié. Il a été souligné que les plans expérimentaux des essais randomisés contrôlés n'étaient pas toujours les plus appropriés pour évaluer l'efficacité des interventions complexes [28,29], notamment en santé publique [47] et en promotion de la santé [48]. En effet, ils permettent difficilement de répondre aux enjeux méthodologiques associés au processus de randomisation, au choix du groupe témoin, aux modifications inopinées 
Tableau 3

Étapes du protocole de l'intervention mapping.

\begin{tabular}{|c|c|c|}
\hline Étapes du protocole & Contenu & Produit \\
\hline $\begin{array}{l}\text { Étape } 1 \\
\text { Évaluation des besoins }\end{array}$ & $\begin{array}{l}\text { Groupe de travail associant les parties prenantes } \\
\text { Évaluation des besoins par indicateurs } \\
\text { Évaluation des besoins dans la perspective des parties }\end{array}$ & $\begin{array}{l}\text { Modèle logique du problème avec les } \\
\text { déterminants des comportements } \\
\text { Formulation des objectifs distaux }\end{array}$ \\
\hline
\end{tabular}

Étape 2

Formulation des objectifs de changement

Étape 3

Choix des cadres théoriques et modalités

Étape 4

Production du programme

Étape 5

Adoption et implantation

Étape 6

Évaluation prenantes

Formulation des objectifs de performance ${ }^{\mathrm{a}}$

Sélection des déterminants importants et modifiables des comportements

Formulation des objectifs de changement ${ }^{\mathrm{a}}$ pour chaque niveau d'intervention

Sélection des cadres théoriques et des techniques de changement pour parvenir aux objectifs de changement Sélection ou conception des applications pratiques

Élaboration de la séquence spatio-temporelle et des éléments matériels du programme

Tests et révision pour améliorer la pertinence culturelle

Fixation des objectifs d'implantation

Identification des barrières et facilitateurs chez les différents acteurs

Élaboration des questions et du plan d'évaluation avec les indicateurs appropriés (processus et résultats)
Matrices des objectifs de changements

Modèle logique théorique du programme justifiant le choix de ses objectifs et des activités pour y parvenir

Modèle logique opérationnel du programme (modèle d'organisation et modèle d'utilisation) Documentation technique, supports d'information, matériels et protocole du programme

Stratégie d'implantation du programme

Étude d'évaluation de l'intervention (implantation, processus, effets)

${ }^{a}$ Les objectifs distaux (finaux) sont les effets attendus du programme sur l'état de santé et/ou la qualité de vie. Les objectifs de performance (intermédiaires) sont les effets attendus du programme sur les comportements dans la population cible et/ou les acteurs de l'environnement. Les objectifs de changement (proximaux) sont les effets attendus du programme sur les déterminants des comportements (connaissances, compétences, efficacité personnelle, norme sociale perçue, attente de résultats, etc.).

de l'intervention sur le terrain, aux tendances séculaires ou aux évolutions du contexte ne pouvant pas être contrôlées. D'autres plans d'études (quasi) expérimentaux (comme les steppedwedge design [49] ou les études avant-après avec groupe témoin) ou observationnels (protocole à niveaux de base multiples, scores de propension, séries chronologiques, etc.) peuvent être choisis selon plusieurs critères comme les caractéristiques des interventions et des populations, les objectifs des différents groupes d'intérêts, le budget alloué, le temps accordé à l'évaluation, etc. [50]. Au-delà des inférences causales sur l'efficacité des interventions (modèle de la boîte noire) [51], la compréhension du mécanisme de ces effets divise la communauté scientifique entre les tenants d'une position épistémologique proche de la philosophie «positiviste » (héritière de la médecine expérimentale) et les tenants de l'approche "réaliste » en évaluation (héritières des sciences sociales) [52-54]. Tous s'accordent cependant sur l'importance de recourir aux théories pour élucider les mécanismes d'action des interventions, et de documenter leurs processus d'implantation. Il est recommandé dans la recherche interventionnelle en santé des populations de recourir à des disciplines et approches variées pour le développement et l'évaluation des programmes de santé [27].

Le programme AMIGAS a fait l'objet d'une évaluation, au moyen d'un essai randomisé contrôlé conduit dans trois bassins de population hispanophone différents qui a montré son efficacité pour augmenter le taux de dépistage (52\% dans le groupe recevant le programme complet contre $25 \%$ dans le groupe témoin) [44], et d'une évaluation économique de type coût-efficacité [55].

Le contenu et les produits des différentes étapes du protocole de l'intervention mapping sont récapitulés dans le Tableau 3.

\section{Discussion}

\subsection{Variété des applications}

Le protocole de l'intervention mapping a été utilisé pour développer et évaluer des interventions dans la plupart des grands domaines de la promotion de la santé. À titre d'exemple, et de façon non exhaustive, on peut citer : la promotion d'une alimentation saine [56], la prévention de l'obésité [57], la promotion de l'activité physique [58], la promotion de la santé sexuelle [59], la prévention des maladies cardio-vasculaires [60], la promotion de la santé mentale [61], la prévention du tabagisme [62], de la consommation excessive d'alcool [63] ou de drogue [64] et la lutte contre le cancer [65].

En promotion de la santé en milieu de travail, des interventions et des outils ont été développés pour promouvoir la reprise du travail après une pathologie musculo-squelettique [66], gynécologique [67], de santé mentale [68] ou après un cancer [69]. D'autres programmes ont visé la prévention des déficits auditifs chez les agriculteurs [70], la promotion de l'activité physique et de la relaxation en entreprise [71], ou la promotion de la vaccination contre la grippe chez les professionnels de santé [72]. 
Ce protocole a été utilisé dans des populations adultes, des populations d'enfants, d'adolescents et d'étudiants, des populations gériatriques, ou encore des populations défavorisées sur le plan socioéconomique ou de la santé mentale.

Le protocole de l'intervention mapping peut être utilisé quel que soit le périmètre d'application, pourvu qu'il entre dans le cadre d'une démarche de planification de la santé. Il a été utilisé à petite échelle dans des écoles [73], des hôpitaux [74] ou des entreprises [75], ou à des échelles plus vastes dans des villes ou des bassins de population [44,76]. À titre d'exemple, le programme d'éducation sexuelle LongLiveLove à l'intention des adolescents a été testé à l'échelle de quelques écoles aux Pays-Bas avant d'être proposé sur tout le territoire [77].

\subsection{Intérêts du protocole}

L'intérêt essentiel du protocole de l'intervention mapping est de fournir aux planificateurs un processus détaillé et structuré pour élaborer, implanter et évaluer des programmes en promotion de la santé. Son degré de précision permet de guider les concepteurs dans la complexité des enjeux méthodologiques et sociaux de cette entreprise. L'approche participative associant des représentants de la population cible et les autres acteurs de l'environnement permet que l'intervention élaborée soit insérée dès le départ dans son contexte d'adoption, augmentant ainsi sa légitimité, sa pertinence et finalement ses chances de pérennité [78]. La vision globale de la santé et de ses déterminants rend justice à la nature sociale des comportements de santé, sans faire porter le poids du changement sur les seuls membres de la population dont les changements de comportement sont visés [2]. Enfin, le recours à des cadres théoriques appropriés en sciences humaines et sociales permet de fonder les programmes sur des bases scientifiques solides, au-delà du sens commun, de l'expérience ou de l'intuition des acteurs. Ces fondements rigoureux permettent d'explorer les chaînes causales associant différents déterminants des problèmes de santé [79], et d'identifier des solutions dont l'efficacité a été démontrée dans la littérature scientifique.

Enfin, les caractéristiques du protocole de l'intervention mapping (vision globale des déterminants de santé, participation des populations visées) et son processus systématisé (formulation du problème, recherche des solutions, implantation, évaluation) en font un outil de choix pour réduire les inégalités sociales de santé en assurant aux interventions développées les critères de qualité recommandés dans ce domaine [80-82]. Il a été utilisé dans cet objectif parmi diverses populations défavorisées [44,83-87].

\subsection{Contraintes d'utilisation}

L'utilisation du protocole de l'intervention mapping présente plusieurs contraintes. Cette démarche de planification tactique et opérationnelle nécessite un minimum de deux à quatre ans selon les études pour être menée à son terme. La constitution d'un groupe de travail collaboratif (comité scientifique élargi) associant des chercheurs et des représentants des différentes parties prenantes est requise, et peut nécessiter du temps et des ressources spécifiques. L'identification de personnes représentatives de la population cible peut se heurter à sa dispersion géographique et à la variété de ses caractéristiques culturelles et sociales. La mobilité géographique de la population cible peut également entraîner des difficultés méthodologiques d'évaluation. Les compétences de recherche doivent être authentiquement pluridisciplinaires, avec la nécessité de compétences spécifiques en psychologie sociale, en psychologie de la santé et dans les sciences du comportement. Ces contraintes nécessitent des financements durables et intersectoriels difficiles à obtenir. L'évaluation critique de l'utilisation du protocole requiert également des compétences éclectiques en recherche, associant l'épidémiologie, les sciences humaines et sociales et la recherche évaluative.

\subsection{Regard critique}

Bien que ce protocole se revendique d'une approche intrinsèquement participative, la lecture critique de quelques programmes publiés soulève plusieurs questions. Certains auteurs invoquant l'utilisation du protocole n'ont pas réalisé les matrices de changement $[88,89]$ alors qu'elles constituent l'étape obligatoire sur laquelle les composantes du programme fondent leur pertinence théorique. Pour d'autres programmes, il est difficile de savoir si une approche participative a été réellement mise en œuvre. Les auteurs décrivent plutôt le développement d'un programme en mode « expert » dans lequel la participation des acteurs impliqués est symbolique ou absente [90]. L'identification des méthodes incorporant dans le programme les identités sociales et culturelles particulières y est rarement explicite. Ces dimensions sont en revanches bien identifiées dans d'autres publications [45,65]. De plus, l'analyse critique des méthodes utilisées pour évaluer les programmes révèle une prédominance presque exclusive des approches expérimentales (essais randomisés simples ou en grappe) et quasi expérimentales (études avant-après avec groupe témoin) au détriment des approches alternatives parfois plus adaptées à la complexité des interventions, des populations visées et de leur contexte. La prudence est donc recommandée avant de considérer le protocole de l'intervention mapping comme une "nouvelle panacée », le risque étant qu'il soit invoqué uniquement dans le discours pour légitimer des entreprises d'imposition d'un idéal normatif dont les paradoxes et les échecs ont été maintes fois soulignés [91]. Cette précaution de principe n'enlève rien à l'intérêt de cette approche complète et structurée dont l'utilisation et les succès auprès de diverses populations soulignent son potentiel de changement.

\section{Conclusion}

Le protocole de l'intervention mapping en promotion de la santé a été utilisé dans de nombreux pays pour de nombreuses problématiques de santé. Cette approche semble particulièrement prometteuse et appropriée aux défis en promotion de la santé, et notamment pour réduire les inégalités sociales de 
santé. Elle reste encore à être développée et testée dans le contexte français, sous réserve que l'approche participative et le recours aux sciences humaines et sociales qui la fondent soient réellement mobilisés. C'est peut-être là son plus grand défi.

\section{Déclaration de liens d'intérêts}

Les auteurs déclarent ne pas avoir de liens d'intérêts.

\section{Références}

[1] Organisation mondiale de la santé. Charte d'Ottawa pour la promotion de la santé. Genève; 1986.

[2] Haut Conseil de la santé publique. Les inégalités sociales de santé : sortir de la fatalité; 2009.

[3] Société française de santé publique. Résultats de la consultation ouverte « Partageons nos expériences pour agir sur les inégalités sociales de santé »; 2014.

[4] Bartholomew K, Parcel G, Kok G, Gottlieb N, Fernandez M. Planning health promotion programs: an intervention mapping approach. 3rd Edition, Sage; 2011.

[5] Bartholomew K, Parcel G, Kok G. Intervention mapping: a process for developing theory - and evidence-based health education programs. Health Educ Behav 1998;25:545-63.

[6] Pommier J, Grimaud O. Les fonctions essentielles de santé publique : histoire, définition et applications possibles. Sante Publique 2007;19: 9-14.

[7] Organisation mondiale de la santé. Glossaire de la promotion de la santé. Genève; 1998.

[8] Bruchon-Schweitzer M, Boujut E. Des croyances aux comportements : conceptions et modèles. Psychologie de la santé. Concepts, méthodes et modèles. $2^{\mathrm{e}}$ édition, Dunod; 2014: 373-432.

[9] Boujut E. Comprendre et prévoir les comportements en santé. Psychologie de la santé. 2e édition, PUF; 2014: 67-88.

[10] Glanz K, Rimer BK, Viswanath K. Health behavior and health education: theory, research and practice. 4th ed. San Francisco: Jossey-Bass; 2008.

[11] Becker MH. The health belief model and personal health behavior. Health Educ Monogr 1974;2:324-508.

[12] Ajzen I. The theory of planned behaviour. Organ Behav Hum 1991;50:179-211.

[13] Bandura A. Self-efficacy: toward a unifying theory of behavioral change. Psychol Rev 1977;84:191-215.

[14] Bandura A. Health promotion by social cognitive means. Health Educ Behav 2004;31:143-64.

[15] Prochaska J, Velicer W. The transtheoretical model of health behavior change. Am J Health Promot 1997;12:38-48.

[16] Weinstein ND, Sandman PM, Blalock SJ. The precaution adoption process model. Health behavior and health education. 4th ed. San Francisco: Jossey-Bass; 2008: 123-47

[17] Jusot F, Tubeuf S, Trannoy A. Circumstances and efforts: how important is their correlation for the measurement of inequality of opportunity in health? Health Econ 2013;22:1470-95.

[18] Trannoy A, Tubeuf S, Jusot F, Devaux M. Inequality of opportunities in health in France: a first pass. Health Econ 2010;19:921-38.

[19] Marmot M, Allen J, Bell R, Bloomer E, Goldblatt P. OMS : un cadre d'action pour réduire les inégalités sociales de santé. La santé de l'homme; 2011: 13-6.

[20] Moleux M, Schaetzel F, Scotton C. Les inégalités sociales de santé : déterminants et modèles d'action. Inspection générale des affaires sociales; 2011.

[21] Aïach P, Baumann M. Prévention et réduction des inégalités de santé : une conciliation difficile. Glob Health Promot 2010;17:95-8.

[22] Pineault R, Daveluy C. La planification de la santé : concepts, méthodes, stratégies. Montréal: Éditions nouvelles; 1995.
[23] Direction de l'hospitalisation et de l'organisation des soins. Cahier des charges pour l'évaluation des besoins de santé dans le cadre de l'élaboration des SROS. Ministère de la santé et de la protection sociale; 2004.

[24] Estellat C. Revue des méthodes pour l'évaluation des besoins de santé. Direction de l'hospitalisation et de l'organisation des soins, Ministère de la santé et de la protection sociale; 2004.

[25] Mackenzie M, O’Donnell C, Halliday E, Sridharan S, Platt S. Do health improvement programmes fit with MRC guidance on evaluating complex interventions? BMJ 2010;340:c185.

[26] Contandriopoulos AP, Champagne F, Denis JL, Avargues MC. L'évaluation dans le domaine de la santé : concepts et méthodes. Rev Epidemiol Sante Publique 2000;48:517-39.

[27] Hawe P, Potvin L. What is population health intervention research? Can J Public Health 2009;100. Suppl I8-14.

[28] Contadriopoulos A-P, Rey L, Brousselle A, Champagne F. Évaluer une intervention complexe : enjeux conceptuels, méthodologiques, et opérationnels. CJPE 2012;26:1-16.

[29] Craig P, Dieppe P, Macintyre S, Mitchie S, Nazareth I, Petticrew M. Developing and evaluating complex interventions: the new Medical Research Council guidance. BMJ 2008;337:979-83.

[30] Greenhalgh T, Robert G, MacFarlane F, Bate P, Kyriakidou O. Diffusion of innovations in service organizations: systematic review and recommendations. Milbank Q 2004;82:581-635.

[31] Rossi PH. Expressing and assessing program theory. Evaluation: a systematic approach. 7th edition, Sage; 2004: 133-68.

[32] Brousselle A, Champagne F, Contadriopoulos A-P, Hartz Z. L'évaluation : concepts et méthodes. $2^{\mathrm{e}}$ ed, Les Presses de 1’Université de Montréal; 2011.

[33] Rogers EM. Diffusion of innovations. 4th ed. New York: The Free Press; 1995.

[34] Damschroder L, Aron D, Keith R, Kirsh S, Alexander J, Lowery J. Fostering implementation of health services research findings into practice: a consolidated framework for advancing implementation science. Implement Sci 2009;4:50.

[35] Fassier JB, Durand MJ, Loisel P. 2nd place, PREMUS best paper competition: implementing return-to-work interventions for workers with low-back pain - a conceptual framework to identify barriers and facilitators. Scand J Work Environ Health 2011;37:99-108.

[36] Fassier JB, Durand MJ, Caillard JF, Roquelaure Y, Loisel P. Results of a feasibility study: barriers and facilitators in implementing the Sherbrooke model in France. Scand J Work Environ Health 2015;41:223-33.

[37] Bartholomew K, Parcel G, Kok G. Intervention mapping: a process for developing theory- and evidence-based health education programs. Health Educ Behav 1998;25:545-63.

[38] Green L, Kreuter M. Health promotion planning: an educational and environmental approach. 1st ed. Mayfield: Mountain View; 1991.

[39] Kotler P, Zaltman G. Social marketing: an approach to planned social change. J Mark 1971;35:3-12.

[40] Chen H-T, Rossi PH. Evaluating with sense: the theory-driven approach. Eval Rev 1983;7:283-302.

[41] Chen H-T, Rossi PH. Issues in the theory-driven perspective. Eval Program Plann 1989;12:299-306.

[42] Pawson R, Tilley A. Realistic evaluation. London: Sage; 1997.

[43] National Cancer Institute. Theory at a glance: a guide for health promotion practice. National Cancer Institute (US department of health and human services); 2005.

[44] Byrd TL, Wilson KM, Smith JL, Coronado G, Vernon SW, FernandezEsquer ME, et al. AMIGAS: a multicity, multicomponent cervical cancer prevention trial among Mexican American women. Cancer 2013;119: $1365-72$.

[45] Byrd TL, Wilson KM, Smith JL, Heckert A, Orians CE, Vernon SW, et al. Using intervention mapping as a participatory strategy: development of a cervical cancer screening intervention for Hispanic women. Health Educ Behav 2012;39:603-11.

[46] Green L, Kreuter M. Health program planning: an educational and ecological approach. 4th ed, McGraw-Hill Higher Education; 2004.

[47] Rychetnik L, Frommer M, Hawe P, Shiell A. Criteria for evaluating evidence on public health interventions [see comment]. J Epidemiol Community Health 2002;56:119-27. 
[48] Tarquinio C, Kivits J, Minary L, Coste J, Alla F. Evaluating complex interventions: perspectives and issues for health behaviour change interventions. Psychol Health 2015;30:35-51.

[49] Hemming K, Haines TP, Chilton PJ, Girling AJ, Lilford RJ. The stepped wedge cluster randomised trial: rationale, design, analysis, and reporting. BMJ 2015;350:h391.

[50] Schelvis RM, Oude Hengel KM, Burdorf A, Blatter BM, Strijk JE, van der Beek AJ. Evaluation of occupational health interventions using a randomized controlled trial: challenges and alternative research designs. Scand J Work Environ Health 2015;41:491-503.

[51] Astbury B, Leeuw FL. Unpacking black boxes: mechanisms and theory building in evaluation. Am J Eval 2010;31:363-81.

[52] Marchal B, Westhorp G, Wong G, Van Belle S, Greenhalgh T, Kegels G, et al. Realist RCTs of complex interventions - an oxymoron. Soc Sci Med 2013;94:124-8.

[53] Bonell C, Fletcher A, Morton M, Lorenc T, Moore L. Methods don't make assumptions, researchers do: a response to Marchal et al. Soc Sci Med 2013;94:81-2.

[54] Blaise P, Marchal B, Lefèvre P, Kegels G, Jones CM. Au-delà des méthodes expérimentales : l'approche réaliste en évaluation. In: Potvin L, Moquet MJ, Jones C, editors. Réduire les inégalités sociales en santé. Institut national de prévention et d'éducation pour la santé; 2010p. 28596.

[55] Lairson DR, Chang YC, Byrd TL, Lee Smith J, Fernandez ME, Wilson KM. Cervical cancer screening with AMIGAS: a cost-effectiveness analysis. Am J Prev Med 2014;46:617-23.

[56] Brug J, te Velde SJ, Chinapaw MJ, Bere E, de Bourdeaudhuij I, Moore H, et al. Evidence-based development of school-based and family-involved prevention of overweight across Europe: the ENERGY-project's design and conceptual framework. BMC Public Health 2010;10:276.

[57] De Henauw S, Verbestel V, Marild S, Barba G, Bammann K, Eiben G, et al. The IDEFICS community-oriented intervention programme: a new model for childhood obesity prevention in Europe? Int $\mathrm{J}$ Obes (Lond) 2011;35(Suppl 1):S16-23.

[58] McKee R, Mutrie N, Crawford F, Green B. Promoting walking to school: results of a quasi-experimental trial. J Epidemiol Community Health 2007;61:818-23.

[59] Aaro LE, Flisher AJ, Kaaya S, Onya H, Fuglesang M, Klepp KI, et al. Promoting sexual and reproductive health in early adolescence in South Africa and Tanzania: development of a theory- and evidence-based intervention programme. Scand J Public Health 2006;34:150-8.

[60] Gillison F, Greaves C, Stathi A, Ramsay R, Bennett P, Taylor G, et al. 'Waste the Waist': the development of an intervention to promote changes in diet and physical activity for people with high cardiovascular risk. Br J Health Psychol 2011;17:327-45.

[61] Cabassa LJ, Druss B, Wang Y, Lewis-Fernandez R. Collaborative planning approach to inform the implementation of a healthcare manager intervention for Hispanics with serious mental illness: a study protocol. Implement Sci 2011;6:80

[62] Cote F, Godin G, Gagne C. Efficiency of an evidence-based intervention to promote and reinforce tobacco abstinence among elementary schoolchildren in a school transition period. Health Educ Behav 2006;33: 747-59.

[63] Voogt CV, Poelen EA, Kleinjan M, Lemmers LA, Engels RC. The development of a web-based brief alcohol intervention in reducing heavy drinking among college students: an intervention mapping approach. Health Promot Int 2013;29:669-79.

[64] Zule WA, Coomes CM, Karg R, Harris JL, Orr A, Wechsberg WM. Using a modified intervention mapping approach to develop and refine a singlesession motivational intervention for methamphetamine-using men who have sex with men. Open AIDS J 2010;4:132-40.

[65] Hou SI, Fernandez ME, Parcel GS. Development of a cervical cancer educational program for Chinese women using intervention mapping. Health Promot Pract 2004;5:80-7.

[66] Ammendolia C, Cassidy D, Steensta I, Soklaridis S, Boyle E, Eng S, et al. Designing a workplace return-to-work program for occupational low back pain: an intervention mapping approach. BMC Musculoskelet Disord 2009;10:65.
[67] Vonk Noordegraaf A, Huirne JA, Pittens CA, van Mechelen W, Broerse JE, Brolmann HA, et al. eHealth program to empower patients in returning to normal activities and work after gynecological surgery: intervention mapping as a useful method for development. J Med Internet Res 2012; $14:$ e124.

[68] van Oostrom SH, Anema JR, Terluin B, Venema A, de Vet HC, van Mechelen W. Development of a workplace intervention for sick-listed employees with stress-related mental disorders: intervention mapping as a useful tool. BMC Health Serv Res 2007;7:127.

[69] Munir F, Kalawsky K, Wallis DJ, Donaldson-Feilder E. Using intervention mapping to develop a work-related guidance tool for those affected by cancer. BMC Public Health 2013;13:6.

[70] Fernandez ME, Bartholomew LK, Alterman T. Planning a multilevel intervention to prevent hearing loss among farmworkers and managers: a systematic approach. J Agric Saf Health 2009;15:49-74.

[71] Coffeng JK, Hendriksen IJ, Duijts SF, Twisk JW, van Mechelen W, Boot CR. Effectiveness of a combined social and physical environmental intervention on presenteeism, absenteeism, work performance, and work engagement in office employees. J Occup Environ Med 2014;56: 258-65.

[72] Riphagen-Dalhuisen J, Frijstein G, van der Geest-Blankert N, DanhofPont M, de Jager H, Bos N, et al. Planning and process evaluation of a multi-faceted influenza vaccination implementation strategy for health care workers in acute health care settings. BMC Infect Dis 2013;13:235.

[73] Beaulieu D, Godin G. Development of an intervention programme to encourage high school students to stay in school for lunch instead of eating at nearby fast-food restaurants. Eval Program Plann 2012;35:382-9.

[74] Schmid AA, Andersen J, Kent T, Williams LS, Damush TM. Using intervention mapping to develop and adapt a secondary stroke prevention program in Veterans Health Administration medical centers. Implement Sci 2010;5:97.

[75] Oude Hengel KM, Joling CI, Proper KI, van der Molen HF, Bongers PM. Intervention mapping as a framework for developing an intervention at the worksite for older construction workers. Am J Health Promot 2011;26:e1-e10.

[76] Hou SI, Fernandez ME, Baumler E, Parcel GS. Effectiveness of an intervention to increase Pap test screening among Chinese women in Taiwan. J Community Health 2002;27:277-90.

[77] Schutte L, Meertens RM, Mevissen FE, Schaalma H, Meijer S, Kok G. Long Live Love. The implementation of a school-based sex-education program in The Netherlands. Health Educ Res 2014;29:583-97.

[78] Pluye P, Potvin L, Denis J-L, Pelletier J, Mannoni C. Program sustainability begins with the first events. Eval Program Plann 2005;28:123-37.

[79] Lang T, Kelly-Irving M, Delpierre C. Inégalités sociales de santé : du modèle épidémiologique à l'intervention. Enchaînements et accumulations au cours de la vie. Rev Epidemiol Sante Publique 2009;57:429-35.

[80] Potvin L, Moquet M-J, Jones CM. Réduire les inégalités sociales en santé. Institut national de prévention et d'éducation pour la santé; 2010.

[81] EuroHealthNet. Questionnaire «Bonnes pratiques dans la lutte contre les inégalités de la santé en Europe ». European partners for equity in health; 2004.

[82] Getting Evidence into Practice - Evidence Consortium. EQUIHP European quality instrument for health promotion. Instrument européen pour la qualité en promotion de la santé; 2005.

[83] Abbema EA, Van Assema P, Kok GJ, De Leeuw E, De Vries NK. Effect evaluation of a comprehensive community intervention aimed at reducing socioeconomic health inequalities in the Netherlands. Health Promot Int 2004;19:141-56.

[84] Batterham RW, Buchbinder R, Beauchamp A, Dodson S, Elsworth GR, Osborne RH. The OPtimising HEalth LIterAcy (Ophelia) process: study protocol for using health literacy profiling and community engagement to create and implement health reform. BMC Public Health 2014;14:694.

[85] Leerlooijer JN, Kok G, Weyusya J, Bos AE, Ruiter RA, Rijsdijk LE, et al. Applying intervention mapping to develop a community-based intervention aimed at improved psychological and social well-being of unmarried teenage mothers in Uganda. Health Educ Res 2014;29:598-610.

[86] Suzuki R, Peterson JJ, Weatherby AV, Buckley DI, Walsh ES, Kailes JI, et al. Using intervention mapping to promote the receipt of clinical 
preventive services among women with physical disabilities. Health Promot Pract 2010;13:106-15.

[87] Van Der Veen YJ, Van Empelen P, Richardus JH. Development of a culturally tailored Internet intervention promoting hepatitis B screening in the Turkish community in the Netherlands. Health Promot Int 2011; 27:342-55.

[88] Vernon SW, Bartholomew LK, McQueen A, Bettencourt JL, Greisinger A, Coan SP, et al. A randomized controlled trial of a tailored interactive computer-delivered intervention to promote colorectal cancer screening: sometimes more is just the same. Ann Behav Med 2011;41:284-99.
[89] Vernon SW, del Junco DJ, Tiro JA, Coan SP, Perz CA, Bastian LA, et al. Promoting regular mammography screening II. Results from a randomized controlled trial in US women veterans. J Natl Cancer Inst 2008; 100:347-58.

[90] Albada A, van Dulmen S, Otten R, Bensing JM, Ausems MG. Development of E-info gene(ca): a website providing computer-tailored information and question prompt prior to breast cancer genetic counseling. J Genet Couns 2009; 18:326-38.

[91] Dozon J-P, Fassin D. Critique de la santé publique. Une approche anthropologique. Paris: Balland; 2001. 